\title{
A Base on Fuzzy Theory to Supplier Evaluation and Selection Optimization
}

\author{
Chun-Tsai Lin \\ Department of Food \& Beverage Management, Cheng Shiu University, Kaohsiung, Taiwan \\ Correspondence should be addressed to Chun-Tsai Lin; capital789@yahoo.com.tw
}

Received 27 July 2020; Revised 9 September 2020; Accepted 23 September 2020; Published 5 October 2020

Academic Editor: Yunqiang Yin

Copyright (C) 2020 Chun-Tsai Lin. This is an open access article distributed under the Creative Commons Attribution License, which permits unrestricted use, distribution, and reproduction in any medium, provided the original work is properly cited.

\begin{abstract}
Supplier evaluation and selection is critical for schools to save costs. Supplier evaluation indexes must be developed before evaluation. In addition, how to select the most appropriate supplier and make the best procurement decision are also important topics. This study selected three most important supplier evaluation and selection criteria and established a supplier evaluation model through fuzzy logic inference using MATLAB. This method can overcome the defects in some methodologies and improve organizational capability and competitiveness. This model can also be used to address practical supplier evaluation and selection matters. Modelling took several steps, including fuzzification, fuzzy rule base, fuzzy inference engine, and defuzzification. Then, a supplier evaluation model was established based on the fuzzy rules obtained from the abovementioned operations. This model can provide a basis for school purchasers or managers in executing supplier selection policies in the future.
\end{abstract}

\section{Introduction}

With increasingly intense market competition and growing consumer awareness over recent years, consumers are provided with more and more opportunities to select the products they need. Mass, simple, and standard production cannot effectively satisfy ever-changing consumer demands any more. Manufacturers must be able to efficiently provide diverse products to satisfy consumer demands and maintain their presence against fierce competition. Schools also represent a group of consumers who frequently procure large amounts of various products. Therefore, supplier selection not only affects a school's procurement cost but also affects its operation quality. Supplier selection is a key step in the procurement process. A school that does not engage an appropriate supplier to provide low-cost and high-quality products cannot attract students, which will invisibly increase costs. This study, based on the data acquired from a supplier database and literature review, used fuzzy logic to propose an evaluation method which addressed such problems. Using a germane supplier selection and evaluation model to cut school procurement cost, it is also the key for schools to increase school competitiveness. Therefore, a supplier evaluation model provides an important basis for school procurement decisions $[1,2]$.

\section{Fuzzy Theory Method}

Fuzzy theory is a famous paper "Fuzzy Sets (Fuzzy Sets), which was published by Professor LA Zadeh of the University of California, Berkeley, in 1965, in the Information and Control (Information and Control) academic journal)." [3]. He believes that traditional control theory places too much emphasis on precision to master complex systems. If you use the concepts of fuzzy sets and continuous membership functions (Membership Function) to control, you won't have this problem. In 1973, he published a paper introducing the concept of semantic variables and proposed the use of fuzzy logic (Fuzzy If-Then Rule) [4] to formulate human knowledge and establish the basis of fuzzy control. In 1978, the first fuzzy controller appeared to verify the feasibility of fuzzy theory [5]. However, the scholars at that time insisted on the traditional set theory, so when the fuzzy theory was put forward, it received many criticisms, but it developed rapidly in the following two decades and combined with other different theories to become a new branch 
of mathematics, and the related applications were also exponential. Growth, such as the application of fuzzy logic systems [6], image recognition [7], time series prediction [8], intelligent robots [9], and its scope, is quite extensive (Figure 1). The basic structure of the fuzzy system is shown in Figures 2 and 3. The main functional blocks include: (1) fuzzification, (2) fuzzy rule base, (3) fuzzy inference engine, and (4) defuzzification.

2.1. Fuzzification and Attribution Function. Fuzzy theory uses the attribute function of the range in the interval $[0,1]$ to represent the fuzzy set on the range U.Common fuzzy attribution functions include trapezoidal and triangular attribution functions.

(1) Triangular attribute function, as shown in Figure 2. The mathematical expression of the triangle attribution function is

$$
\mu_{A}(x)= \begin{cases}\frac{x-k}{m-k}, & k \leq x \leq n, \\ \frac{r-x}{r-m}, & m<x \leq r, \\ 0, & \text { otherwise. }\end{cases}
$$

(2) Trapezoidal attribute function, as shown in Figure 3.

The mathematical expression of the trapezoidal attribute function is

$$
\mu_{A}(x)=\left\{\begin{array}{lc}
\frac{x-k}{a-k}, & k \leq x \leq a, \\
1, & a \leq x \leq b, \\
\frac{r-x}{r-b}, & b<x \leq r, \\
0, & \text { otherwise. }
\end{array}\right.
$$

2.2. Fuzzy Rule Base. Fuzzy Rule Base is a classification rule of fuzzy theory. The design method is expressed in the form of the If-Then column formula, and the fuzzy control algorithm is shown in the following formula:

$$
\begin{aligned}
& \text { If }\left\langle x_{1}\right\rangle \text { is }\left\langle A_{1}\right\rangle \text { and } \ldots \text { and }\left\langle x_{i}\right\rangle \text { is }\left\langle A_{i}\right\rangle \quad i=1,2, \ldots, n \text {, } \\
& \text { Then } y \text { is }\left\langle\text { action }_{i}\right\rangle \text {, }
\end{aligned}
$$

where $n$ is the number of control rules, $x$ is the input parameter, $A$ is the condition, and action is the corresponding action based on the previous conditions. Generally speaking, there are three main methods for generating rule bases:

(1) Based on expert knowledge and experience: a fuzzy rule library is designed based on expert consultation

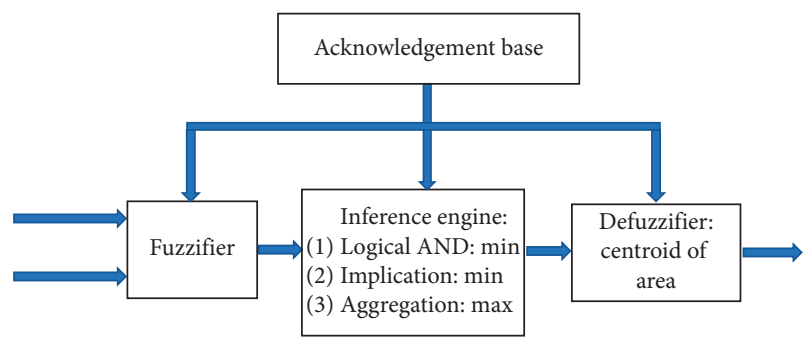

Figure 1: Fuzzy inference.

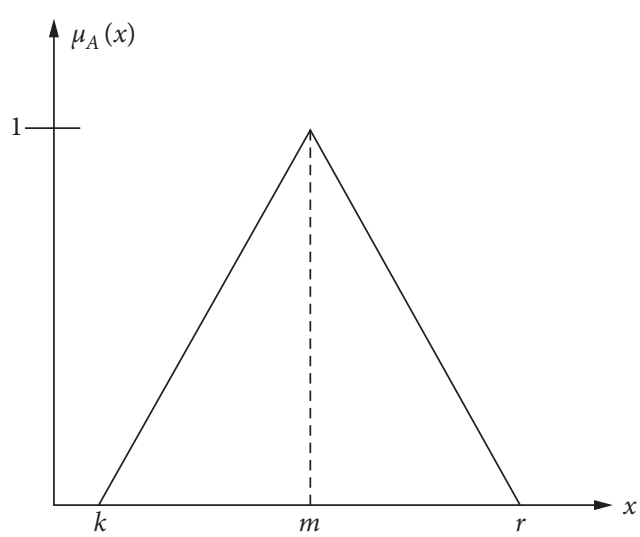

Figure 2: Triangle attribute function.

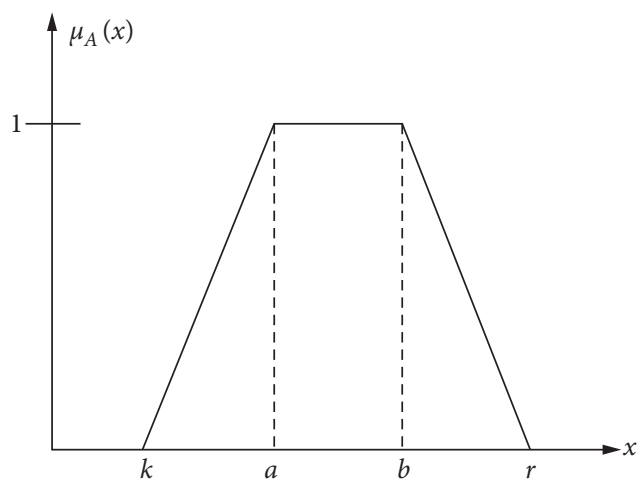

FIgURE 3: Trapezoidal attribution function.

with experience in this research field or reference books with reference value.

(2) Response according to the input of the controlled object: according to the input and output reaction actions of the controlled object, it can be summarized as the method of system identification.

(3) Through self-learning: after collecting some measurement data, the data are measured by a specific training algorithm, and fuzzy rules are extracted from it, for example, Genetic Algorithm (GA) and Artificial Neural Network (ANN).

2.3. Fuzzy Inference Engine. The fuzzy inference engine is the core of the fuzzy system. It simulates human thinking and decision-making modes through approximate inference or 


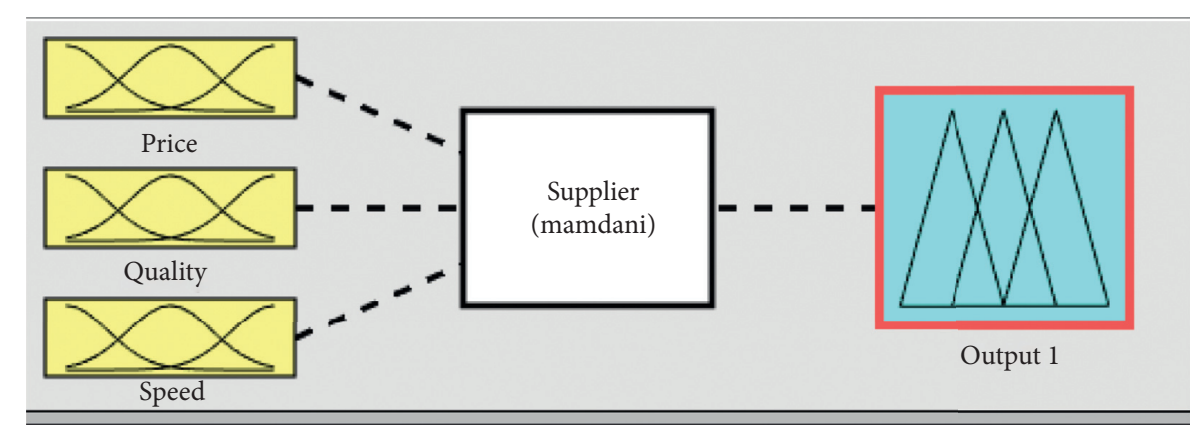

FIGURE 4: Fuzzy inference system.

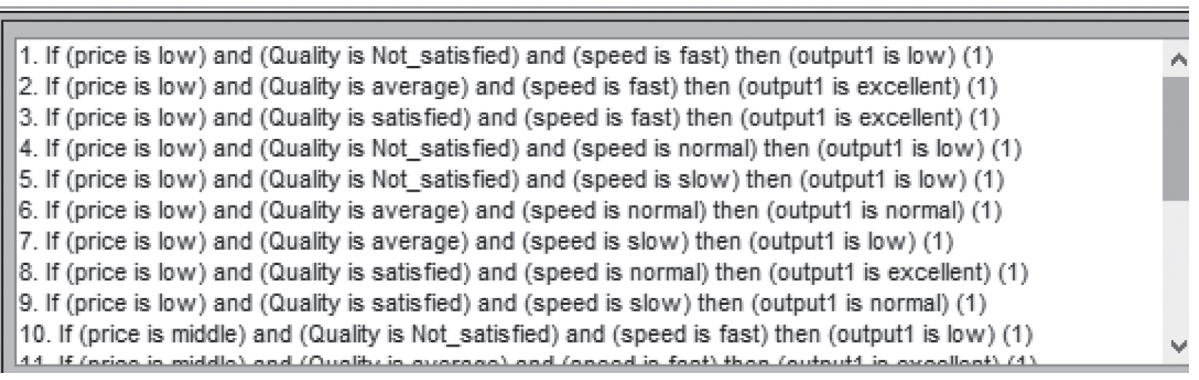

Figure 5: Fuzzy rules.

fuzzy inference to achieve the main purpose of problem solving. In this research, the maximum-minimum composite operation in fuzzy inference is used. The formula is as follows:

Maximum-minimum composite operation:

$$
\mu_{C^{\prime}}(z)=\max _{x}\left[\min \left(\mu_{A}(x), \mu_{A \longrightarrow B}(x, y)\right)\right] .
$$

2.4. Defuzzification. After the parameters are fuzzified and fuzzy inference engine, clear values must be solved through defuzzification. The process of converting the conclusions generated after fuzzy inference into clear values is called "defuzzification." The legal meaning is to calculate the area center point of the overlapping area, and the set value of the position of the center of gravity point is the fuzzy output value as shown in the following formula:

$$
y^{*}=\frac{\left[\sum_{i=1}^{N} \mu_{C}\left(y_{i}\right) \cdot y_{i}\right]}{\sum_{i=1}^{N} \mu_{C}\left(y_{i}\right)} .
$$

Among them, $N$ is the total number of fuzzy rules, $y_{i}$ is the inferred result of the $i$-th fuzzy rule, $\mu_{C}\left(y_{i}\right)$ represents the attribution value of the $i$-th rule fuzzy set.

\section{Simulation Results}

This research is based on the supplier data established over the years as a database. Three variables are selected including price, delivery speed, and quality variables. Based on this, a set of fuzzy rules is compiled, and the design steps are as follows:
(1) Input and output language variables are created

(2) Relevant terms are established, and attribution functions are defined

(3) Fuzzy rules and inferences are given

(4) Deblurring

This study designed and constructed a supplier evaluation model based on this rule.

3.1. Creating Input and Output Language Variables. This research uses fuzzy logic inference tools to establish a supplier system and uses fuzzy algorithms to make judgments. Before making judgments, first, the input and output parameters are confirmed, and price, delivery date, and quality are choosen as input parameters.

\subsection{Establishing Related Terms and Attribution Functions.} In the fuzzy judgment, the relevant terms are corresponding to the input language variables, and the input and output settings of each parameter are as follows:

We enter the parameters of price and quality in three steps of low, medium, and high and the delivery speed in three steps of fast, normal, and delayed. After establishing the relevant terminology, an attribution function must be defined for each term. The input and output functions of this study use triangular attribution functions. The various central values of the attribution function and the size of its range will affect the fuzzy value of the input variable, which is good for fuzzy judgment design. The influence is very large, and the suitable value range designed in this study is shown in Figures 4 and 5. 


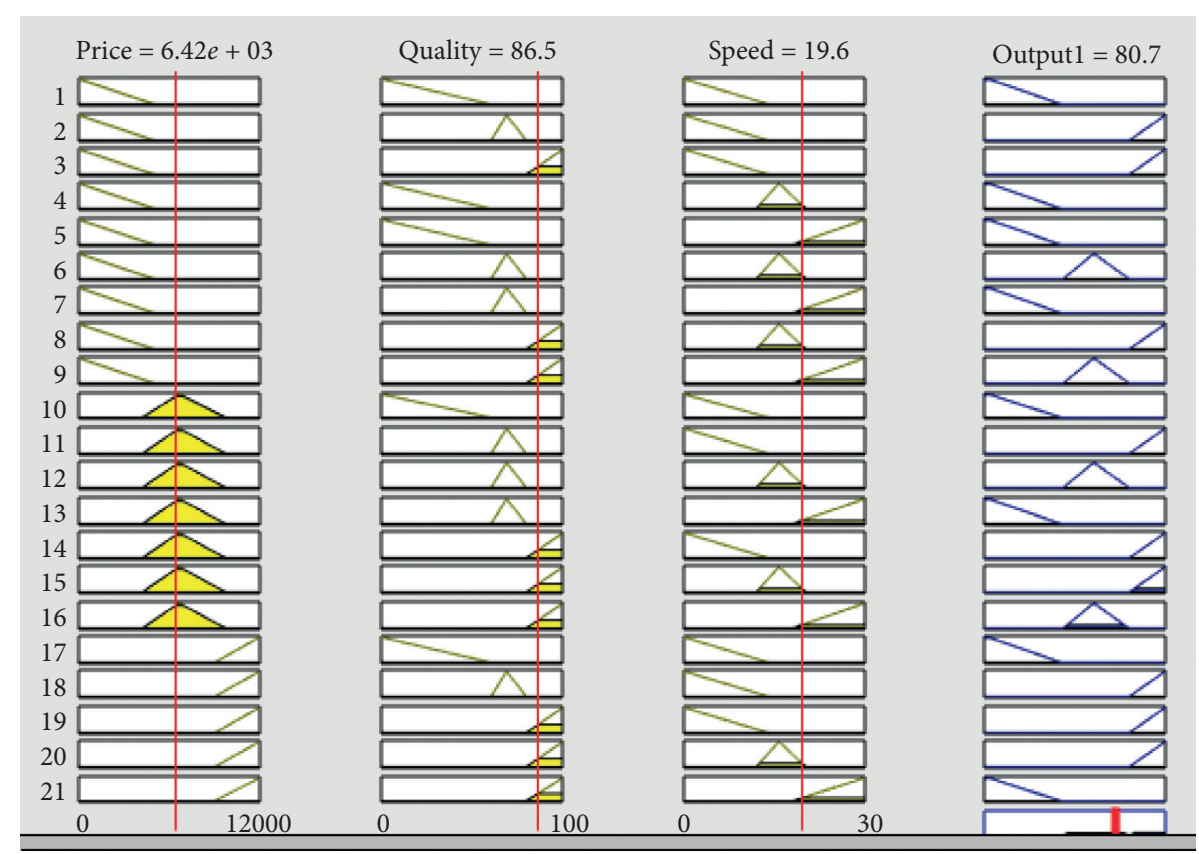

FIgure 6: Fuzzy inference.

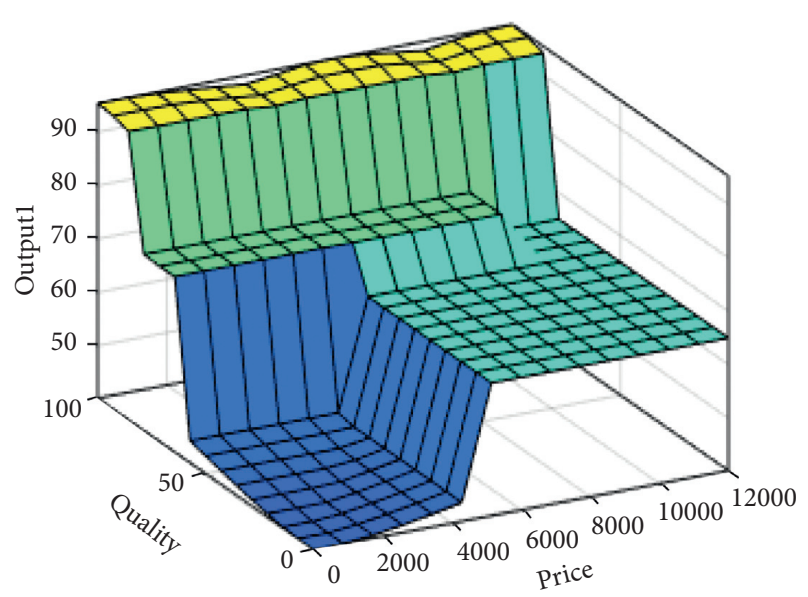

FIgURE 7: Surface plot.

\section{Conclusions}

This study acquired data from a database containing years of supplier data and carried out fuzzy inference and defuzzification using MATLAB. The centroid method was used for defuzzification. As inferenced by it, 27 rules were triggered in total. As shown in the figure, when the price is above NT\$ 5000 , quality is above medium and high levels, delivery is efficient within 20 days, and $80.7 \%$ of suppliers are reliable, as shown in Figures 6 and 7.

Supplier selection is the first step to establish a supplier system. Selection of appropriate suppliers is helpful to school procurement evaluation. In contrast, if inappropriate or uncooperative suppliers are selected, schools (and even their operations) will be negatively affected. Therefore, cautions must be exercised in selecting suppliers. The fuzzy multiple criteria method was used for analyzing and validating the efficacy and feasibility of the model.

\section{Data Availability}

After the article is published and accepted, data sharing and use can be allowed.

\section{Conflicts of Interest}

The authors declare that they have no conflicts of interest.

\section{Acknowledgments}

This work was supported in part by Cheng Shiu University.

\section{References}

[1] A. Brun and A. P. Staudacher, "Negotiation-driven supplyChain Co-ordination small and medium enterprises," in Proceedings of the ECAI 2000 Workshop "13-gent Technologies and their Application Scenarios in Logistics," I. J. Timm, Ed., pp. 55-60, Berlin, Germany, July 2000.

[2] M. Wouters, J. C. Anderson, and F. Wynstra, "The adoption of total cost of ownership for sourcing decisions-a structural equations analysis," Accounting, Organizations and Society, vol. 30, no. 2, pp. 167-191, 2005.

[3] L. A. Zadeh, "Fuzzy sets," Information and Control, vol. 8, no. 3, pp. 338-353, 1965.

[4] L. A. Zadeh, "Outline of a new approach to the analysis of complex systems and decision processes," IEEE Transactions on Systems, Man, and Cybernetics, vol. SMC-3, no. 1, pp. 28-44, 1973.

[5] W. Li-Xin, A Course in Fuzzy Systems and Control, Prentice Hall, Upper Saddle River, NJ, USA, 1997.

[6] T. Munakata and Y. Jani, "Fuzzy systems: an overview," Communications of the ACM, vol. 37, pp. 69-96, 1994. 
[7] J. C. Bezdek, Pattern Recognition with Fuzzy Oßjective Function Algorithms, Springer Science \& Business Media, Berlin, Germany, 2013.

[8] A. Pouraßdollah, C. Wagner, J. H. Aladi, and J. M. Garißaldi, "Improved uncertainty capture for nonsingleton fuzzy systems," IEEE Transactions on Fuzzy Systems, vol. 24, no. 6, pp. 1513-1524, 2016.

[9] Y. P. Kondratenko, O. V. Kozlov, O. S. Gerasin, and Y. M. Zaporozhets, "Synthesis and research of neuro-fuzzy o $\beta$ server of clamping force for mo $\beta$ ile ro $\beta$ ot automatic control system," in Proceedings of the 2016 IEEE First International Conference on Data Stream Mining \& Processing (DSMP), pp. 90-95, Lviv, Ukraine, August 2016. 